\title{
Fourth Branchial Fistula: Revisited
}

\author{
${ }^{1}$ Anandabrata Bose, ${ }^{2}$ Arup Sengupta, ${ }^{3}$ Tshering Dorjee Sherpa, ${ }^{4}$ Nomi Paul
}

\begin{abstract}
Third or fourth branchial pouch anomaly is rare. It usually presents as an acute inflammatory swelling of the lower neck and frequently involves the thyroid gland. Surgical treatment is essential to prevent recurrence. USG, CT scan and barium esophagography help in the diagnosis. Complete excision of tract after proper identification is necessary to prevent recurrence.
\end{abstract}

Keywords: Feeding gastrostomy, Fourth branchial fistula, Pyriform sinus fistula.

How to cite this article: Bose A, Sengupta A, Sherpa TD, Paul N. Fourth Branchial Fistula: Revisited. Int J Head Neck Surg 2015;6(1):26-28.

Source of support: Nil

Conflict of interest: None

\section{CASE REPORTS}

\section{Case 1}

Eight-year-old male child presented with left-sided discharging neck sinus adjacent to the thyroid area of 8 months duration in June 2001. The child was mentally challenged with craniosynostosis which had been operated earlier. He underwent an excision of tract along with a cuff of thyroid tissue soon after. On November 2001, he developed a neck abscess requiring drainage. Computed tomography (CT) scan at the time showed an abscess cavity adjacent to left thyroid lobe with gas shadow (Fig. 1). Hypopharyngoscopy was technically difficult and did not reveal a fistula in PFS. In Jan 2003, he underwent another attempt at excision elsewhere. At the time of operation, a tract was seen going up to esophagus. Six months later, he again developed a fistula with drainage of saliva. A barium esophagogram showed a communication with pyrifrom fossa (Fig. 2). A month later, he underwent yet another excision at another hospital where they felt that the tract was going up to laryngopharynx. Four years later in August 2007, he developed another neck abscess and again on Jan 2010.

\footnotetext{
${ }^{1,2}$ Consultant, ${ }^{3}$ Senior Register, ${ }^{4}$ Register

${ }^{1-4}$ Department of ENT and Head and Neck Surgery, Neotia Getwel Healthcare Center, Siliguri, West Bengal, India

Corresponding Author: Anandabrata Bose, Consultant Department of ENT and Head and Neck Surgery, Neotia Getwel Healthcare Center, Siliguri, West Bengal, India, Phone: 09434007982, e-mail: anandabrata.bose@gmail.com
}

In Feb 2010, we re-excised the tract and the left thyroid lobe with preservation of the left recurrent laryngeal nerve (RLN). Fistulous communication was seen. But, he developed a salivary leak and a barium showed the dye coming out below the left thyroid ala. A percutaneous endoscopic gastrostomy (PEG) was done, and a muscle flap applied to the region. He was kept nil orally for 3 months and repeated barium screening was done. $\mathrm{He}$ started oral feeding without leak thereafter, and the PEG was removed on June 2011. He is asymptomatic till date.

\section{Case 2}

A nine-year-old male child presented with a tenderness and neck stiffness for 2 weeks. He had undergone a left pharyngocoele operation at another institute 2 years earlier. An ultrasonography (USG) showed an abscess but barium swallow did not reveal a leak. He underwent neck abscess drainage and a PEG insertion on July 2010. He developed an intraperitoneal bleed a week later and underwent a laparotomy to stop bleeding from the gastrostomy site. On Feb 2011, he underwent a formal excision of tract and left thyroid lobe with preservation of left RLN. By probing the left pyriform fossa pit with an ureteral catheter (Fig. 3), the internal opening of the tract was identified and ligated (Fig. 4). He was kept nil orally for 3 months and allowed to swallow normally thereafter. He has since been symptom free.

\section{Case 3}

An eleven-year-old male child presented with recurrent left neck abscess. A formal excision of tract along with left thyroid lobe was done on June 2011. At the time of operation, similar probing of the left pyriform fossa pit helped in definite identification and ligation of the tract. Left RLN was preserved. He was kept on nasogastric feeding and allowed to swallow after 2 weeks. He has had no further neck infection since.

\section{DISCUSSION}

Branchial cleft anomalies pertaining to third and fourth arches are rare. Third and fourth branchial arch fistulas have an internal opening at or below the pyriform fossa, hence alluded to as pyriform fossa fistula. A third arch fistula tract should ascends caudal to the glossopharyngeal nerve over the superior laryngeal nerve, posteromedial to 


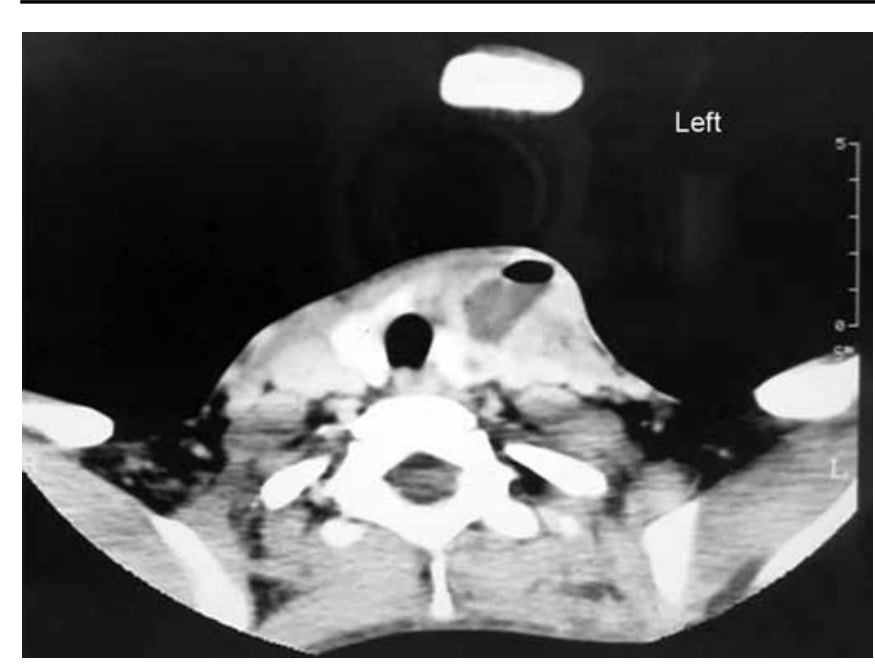

Fig. 1: Computed tomography scan of the neck

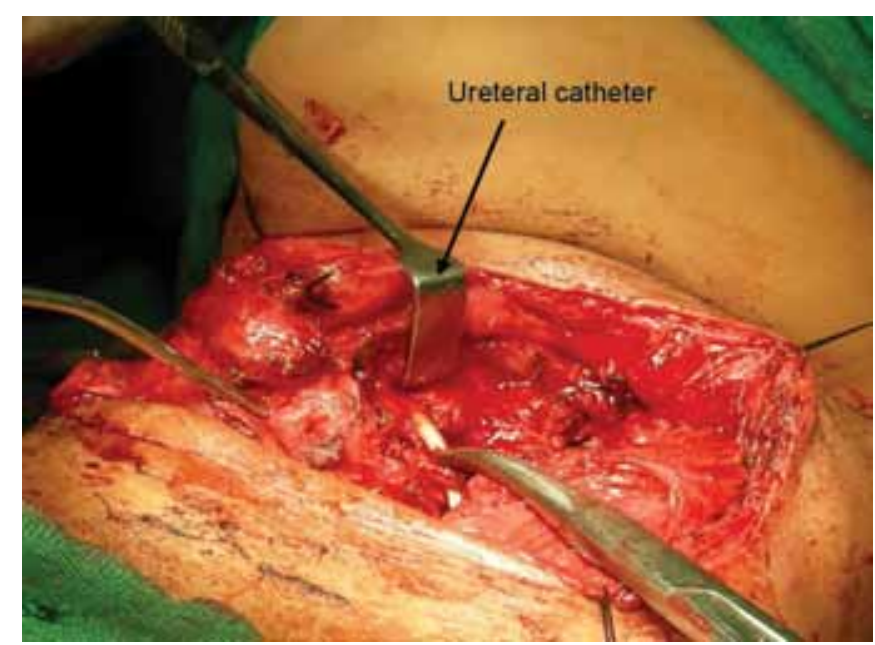

Fig. 3: Catheter probing

internal carotid artery to finally open onto the pyriform fossa. ${ }^{1}$ This does not correlate with surgical findings. The theory of fourth arch fistula due to persistence of the ultimobranchial duct seems more probable. According to this theory, the thymus, before fusion in the midline, retains a lumen called the thymopharyngeal duct. After the fusion of the thymus, this duct usually closes and the thymus then descends at 7 to 8 weeks of gestation. If the thymopharyngeal duct fails to close, its tract would lead from the pyriform fossa through the thyroid gland and then descend to end at the cervical inlet. ${ }^{2}$ In the two cases that we were able to delineate the tract, it was not posterior to the internal carotid artery and intimately related to the thyroid gland superior pole. A further proof would have been if by immunohistochemistry we were able to demonstrate ultimobranchial body. ${ }^{3}$

The presentation in all our cases has been similar as reported in the literature namely recurrent neck abscess. The presentation of the fistula at a slightly older age may be due to increase in swallowing pressure as the child's food quality changes to adult solid food. The fistula usually occurs on the left side as during normal embryo-

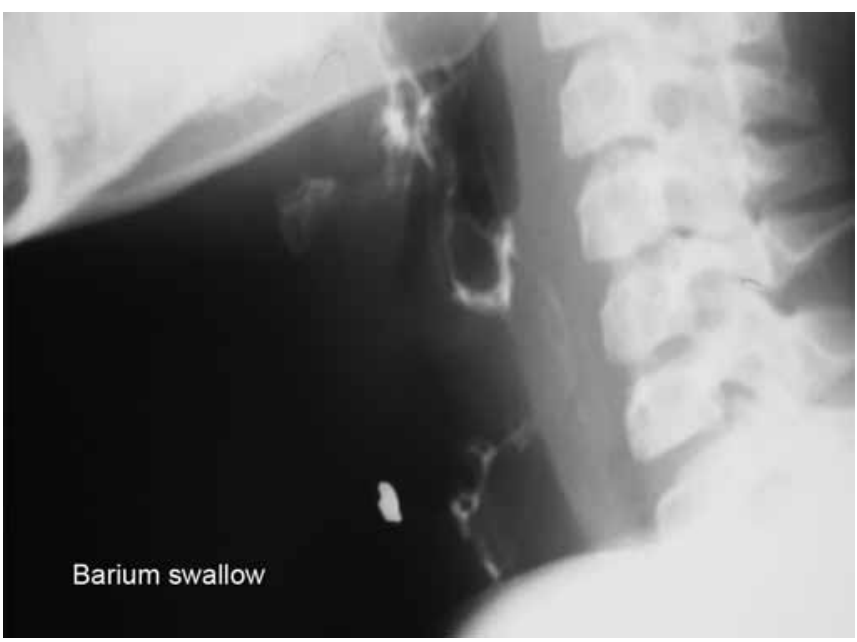

Fig. 2: Barium esophagogram

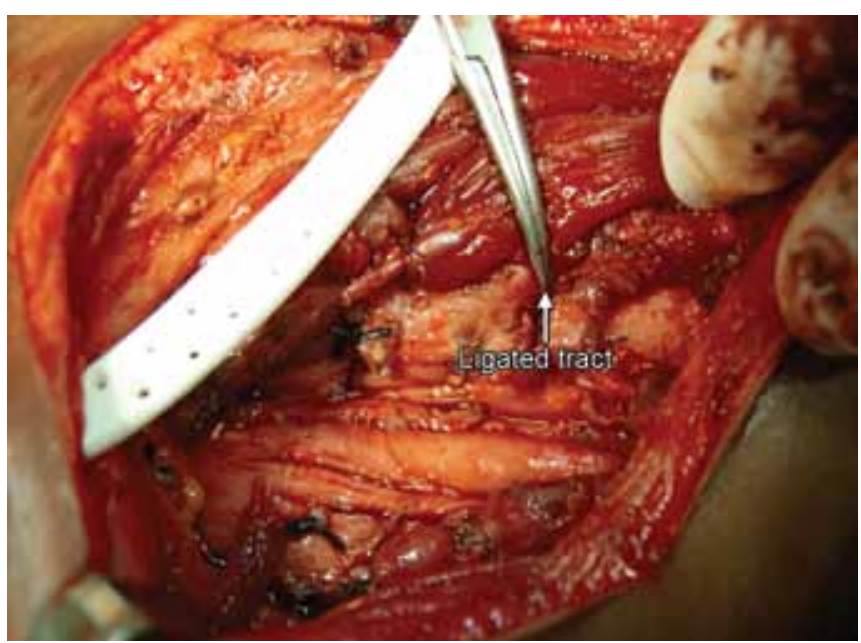

Fig. 4: Ligated branchial tract

logic development of the branchial apparatus, the fourth arch artery on the left side becomes part of the aortic arch, and the fourth arch artery on the right side becomes the proximal portion of the right subclavian artery. This asymmetric development of the branchial apparatus may explain the left-sided predominance of pyriform sinus fistula. ${ }^{4}$

A cost-effective diagnostic tool in our settings is an USG during the acute phase. It shows an abscess with gas shadows and perithyroid edema. The presence of gas shadows would indicate a communication with the viscous nearby. Barium swallow delineates the fistula in the nonacute phase. In the acute phase due to edema and scarring, the fistula opening may not remain patent. Thin barium is advisable. Maneuvers, such as drinking of carbonated beverage prior to the procedure, trumpet blowing or a thin barium swallow prior to scan have been tried to increase the yield of demonstrating a fistula on $\mathrm{CT}^{5}$

In recurrent cases, we have put a PEG which was acceptable to both the children and enabled us to maintain adequate enteral nutrition during the whole period when 
they were kept nil orally. As the tube was under their clothing, they were able to get back to school and play with other kids.

During surgery, identification and ligation of the tract is essential. The recurrence rate can be as high as $22 \%$ specially if there has been previous infection and surgery. ${ }^{6}$ Though methylene blue has been advocated in the presence of an external fistula, we have found it not suitable in our cases. ${ }^{7}$ The intense scarring from repeated infection makes it difficult to get a tract for cannulation or injection from the external side. Probing the internal opening in the pyriform fossa is also challenging. We have tried red rubber catheter and Fogarty catheter but they are extremely pliable and difficult to negotiate through the endoscope. ${ }^{7}$ In our experience, a ureteral catheter has been most useful as it is thin, has pliability as well as rigidity required for the purpose. We believe removing the thyroid lobe helps in complete excision of tract, better identification with preservation of the recurrent laryngeal nerve and reduces recurrence as has been suggested by other authors. ${ }^{8}$ Moreover, the posterior plane of dissection is not violated by the superficial abscess drainage procedures and, hence, easier to dissect as we have seen in our last patient.

\section{CONCLUSION}

The awareness about this rare entity will help us in suspecting a branchial fistula in recurrent lower neck abscess adjacent or involving the thyroid gland. The presence of gas on USG will further affirm the presence of a communication with the food passage. Barium esophagogram may show a fistula in the nonacute phase. Removal of thyroid gland will help to completely excise tract after proper delineation with endoscopic probing with suitable material and save the recurrent laryngeal nerve as well. This will help in preventing recurrence. Long-term follow-up is necessary to ascertain the success of the procedure.

\section{REFERENCES}

1. Cases JA, Wenig BM, Silver CE, et al. Recurrent acute suppurative thyroiditis in an adult due to fourth branchial pouch fistula. J Clin Endocrinol and Metabolism 2000;85(3):953-956.

2. James A, Stewart C, Warrick P, Tzifa C, Forte V. Branchial sinus of the piriform fossa: reappraisal of third and fourth branchial anomalies. Laryngoscope 2007;117(11):1920-1924.

3. Miyauchi A, Matsuzuka F, Kuma K, Katayama S. Piriform sinus fistula and the ultimobranchial body. Histopathology 1992;20(3):221-227.

4. Wang HK, Tiu CM, Chou YH, Chang CY. Imaging studies of pyriform sinus fistula. Pediatr Radiol 2003;33(5):328-333.

5. Gan YU, Lam SL. Imaging findings in acute neck infection due to pyriform sinus fistula. Ann Acad Med Singapore 2004; 33(5):636-640.

6. Choi SS, Zalzal GH. Branchial anomalies: a review of 52 cases. Laryngoscope 1995;105(9):909-913.

7. Aneeza WH, Mazita A, Marina MB, Razif MY. Complete congenital third branchial fistula: does the theoretical course apply? Singapore Med J 2010;51(7):e122-e125.

8. Yang C, Cohen J, Everts E, et al. Fourth branchial arch sinus: clinical presentation, diagnostic workup, and surgical treatment. Laryngoscope 1999;109(3):442-446. 